\title{
Manifestações dos distúrbios hipertensivos da gravidez e complicações associadas à infecção por COVID-19
}

\author{
Manifestations of hypertensive disorders of pregnancy and complications associated with \\ COVID-19 infection
}

\section{Manifestaciones de trastornos hipertensivos del embarazo y complicaciones asociadas con la infección por COVID-19}

Caislane Maria Santos Morais ${ }^{1 *}$, Amanda Otoni Duarte ${ }^{2}$, Anne Caroline Rogienfisz Mendes ${ }^{3}$, Beatriz Barreto de Andrade ${ }^{1}$, Isabella Martins Brito ${ }^{4}$, Isadora Campos de Oliveira ${ }^{5}$, Laís Maria Borges Marins $^{6}$, Letícia Farias Oliveira ${ }^{7}$, Matheus Silva Meireles Ferreira ${ }^{7}$, Maria Luísa Barros Quintão Couto Parreira ${ }^{8}$.

\section{RESUMO}

Objetivo: Descrever e discutir as manifestações da doença hipertensiva específica da gravidez (DHEG) e a possível relação dessa complicação gestacional com o cenário atual da pandemia do COVID-19. Revisão bibliográfica: As principais causas de morte materna e perinatal são as síndromes hipertensivas gestacionais e grande parte desses casos podem ser evitados com o diagnóstico e o tratamento estabelecidos precocemente. Apesar da etiologia da DHEG não ser bem estabelecida, sabe-se que há relação com distúrbios placentários, lesão endotelial e inflamação generalizada. Esses fatores podem ser agravados perante infecção por COVID-19, visto que há interação desse vírus com a expressão de enzimas envolvidas na regulação da pressão arterial, além da relação com o aumento de distúrbios tromboembólicos. Considerações finais: $O$ presente trabalho mostrou a importância da detecção e tratamento precoce da DHEG, bem como do cuidado quanto à infecção das gestantes pelo COVID-19 no atual momento de pandemia. Além disso, reforça-se a necessidade que as equipes de saúde planejem a melhor forma de assistência pré-natal neste período, considerando a telemedicina sempre que sua presença física puder ser evitada no consultório médico.

Palavras-chave: Hipertensão induzida pela gravidez, Síndrome HELLP, Infecções por coronavírus.

\begin{abstract}
Objective: Describe and discuss the manifestations of hypertensive disorders of pregnancy (DHEG) and the possible connexion of this gestational complication with the present pandemic scene of COVID-19. Bibliographic review: The main causes of maternal and perinatal death are gestational hypertensive syndromes and most of these cases can be avoided with early diagnosis and treatment. Although the etiology of DHEG is not well established, it is known that there is a relationship with placental anomaly, endothelial injury and generalized inflammation. These factors can be aggravated by infection with COVID-19, since there is an interaction of this virus with the expression of enzymes involved in steady blood pressure, further on the relationship with the increase in thromboembolic disorders. Final considerations: The present study showed the importance of the early detection and treatment of DHEG, as well as the care regarding the infection of pregnant women by COVID-19 in the current moment of the pandemic. Furthermore, it reinforces the need for health teams to plan the best form of prenatal care during this period, considering telemedicine whenever their physical presence can be avoided in the doctor's clinic.
\end{abstract}

Key words: Hypertension pregnancy induced, HELLP syndrome, Coronavirus infections.

\footnotetext{
${ }^{1}$ Faculdade Estácio, Alagoinhas - BA. *E-mail: kaislane_sf@hotmail.com

2 Centro Universitário de Caratinga (UNEC), Caratinga - MG.

${ }^{3}$ Universidade Fundação Osvaldo Aranha (UniFOA), Volta Redonda - RJ.

${ }^{4}$ Centro Universitário de Belo Horizonte (UniBH), Belo Horizonte - MG.

5 Universidade de Rio Verde (UniRV), Formosa - GO.

${ }^{6}$ Centro Universitário do Planalto Central Apparecido dos Santos (Uniceplac), Gama - DF.

${ }^{7}$ Centro Universitário Uninovafapi (UNINOVAFAPI), Teresina - PI.

${ }^{8}$ Faculdade de Medicina do Vale do Aço (UNIVAÇO), Ipatinga - MG.
} 


\section{RESUMEN}

Objetivo: Describir y discutir las manifestaciones de los trastornos hipertensivos del embarazo (HIE) y la posible relación de esta complicación gestacional con el escenario actual de la pandemia de COVID-19. Revisión bibliográfica: Las principales causas de muerte materna y perinatal son los síndromes hipertensivos gestacionales y la mayoría de estos casos pueden evitarse con un diagnóstico y tratamiento precoces. Aunque la etiología de la HIE no está bien establecida, se sabe que existe una relación con trastornos placentarios, lesión endotelial y inflamación generalizada. Estos factores pueden verse agravados por la infección por COVID-19, ya que existe una interacción de este virus con la expresión de enzimas involucradas en la regulación de la presión arterial, además de la relación con el aumento de trastornos tromboembólicos. Consideraciones finales: El presente estudio mostró la importancia de la detección precoz y el tratamiento de la HIE, así como la atención en cuanto a la infección de las embarazadas por COVID-19 en el momento actual de la pandemia. Además, refuerza la necesidad de que los equipos de salud planifiquen la mejor forma de atención prenatal durante este período, considerando la telemedicina siempre que se pueda evitar su presencia física en el consultorio médico.

Palabras clave: Hipertensión inducida en el embarazo, Síndrome HELLP, Infecciones por coronavirus.

\section{INTRODUÇÃO}

A Doença Hipertensiva Específica Da Gravidez (DHEG) constitui uma das principais causas de mortalidade materna e perinatal em todo o mundo, com incidência em 7 a 10\% de todas as gestações, com variações conforme a população estudada ou metodologia utilizada. Na Ásia e na África, quase um décimo de todas as mortes maternas está associado à DHEG e, na América Latina, essas complicações podem ser responsáveis por até 25\% dos óbitos maternos (MÉHATS C, et al., 2017; OMS, 2014).

Para efeitos de classificação, considera-se como hipertensão crônica aquela alteração pressórica observada antes da gravidez, ou antes de 20 semanas de gestação (pressão arterial sistólica maior ou igual a $140 \mathrm{mmHg}$ e/ou pressão arterial diastólica maior ou igual a $90 \mathrm{mmHg}$ ). A pré-eclâmpsia (PE), por sua vez, é quando a hipertensão arterial (HA) é identificada pela primeira vez após a vigésima semana de gestação, frequentemente acompanhada de proteinúria. Entretanto, segundo o American College of Obstetricians and Gynecologists (ACOG) (2020), a hipertensão e outros sinais ou sintomas de PE podem se apresentar em algumas mulheres na ausência de proteinúria. Já a eclâmpsia é caracterizada pela presença de convulsões tônico-clônicas generalizadas ou coma em mulher com qualquer quadro hipertensivo, não causadas por epilepsia ou qualquer outra doença convulsiva, seja na gravidez, parto ou puerpério imediato (FEBRASGO, 2017; ACOG, 2020).

Sabe-se que a patogênese da PE se relaciona a distúrbios placentários no começo da gravidez, além da presença de inflamação generalizada e lesão endotelial progressiva. Estudos epidemiológicos demonstram, ainda, que a Síndrome HELLP, que é caracterizada pela hemólise, níveis elevados de enzimas hepáticas e baixa contagem de plaquetas, ocorre em $10 \%$ a $20 \%$ das mulheres com pré-eclâmpsia grave, e implica em até $5 \%$ de óbito materno e 60\% de mortalidade perinatal (RIBEIRO JF, et al., 2017; FEBRASGO, 2017; OMS, 2014).

Os fatores de risco da hipertensão gestacional estão atrelados ao consumo excessivo de sódio, antecedentes familiares, diabetes, obesidade, hipotireoidismo e sedentarismo. Em virtude dos mecanismos fisiopatológicos que comprometem a gestação, o diagnóstico de DHEG prévio ou estabelecido até a 20aㅗ semana reflete no perfil de prognóstico materno fetal (BROWN AM, et al., 2018). Dessa forma, em quadros da hipertensão avançada, observam-se complicações como o abortamento, parto prematuro, restrição do crescimento fetal, descolamento da placenta, sofrimento fetal e afecções em órgãos vitais após o nascimento (SOUSA MG, et al., 2020)

Atualmente, o cenário da pandemia ocasionada pelo coronavírus 2 (SARS- COV-2) sugere aumento de casos de PE, que podem estar associados ao mecanismo de infecção do vírus que resulta em vasoconstrição e lesão endotelial. Da mesma forma, a PE envolve estresse oxidativo e estado pró trombótico, implicando na gênese de danos endoteliais. Logo, relatórios iniciais sugerem taxas mais altas de PE e outras complicações 
relacionadas à gravidez com infecção por SARS-CoV-2, uma vez que COVID-19 e a PE, quando sobrepostas, intensificam desordens sistêmica tais como hipertensão, insuficiência renal e hepática e trombocitopenias, por compartilharem aspectos fisiopatológicos semelhantes. Dessa forma, é urgente a necessidade de pesquisas sobre o tratamento ideal para COVID-19 e estratégias preventivas durante a gravidez (MENDOZA M, et al., 2020; KARIMI L, et al., 2020; NARANG K, et al., 2020).

Diante desse contexto, o objetivo do presente trabalho é, a partir da revisão da literatura atual, descrever e discutir as manifestações da DHEG e a possível relação dessa complicação gestacional com o cenário atual da pandemia do COVID-19.

\section{REVISÃO BIBLIOGRÁFICA}

O período gestacional é marcado por diversas modificações uteroplacentárias e metabólicas a fim de proporcionar a vitalidade do feto em crescimento, entretanto, essas alterações fisiológicas predispõem a riscos significativos durante todo o período gestacional. A implantação placentária, por exemplo, pode interferir na redistribuição do fluxo sanguíneo que, associado à alterações imunológicas na gestação, interferem na resposta vascular e isso, implica em limitações ao fluxo sanguíneo da placenta. Por conseguinte, pode ocorrer lesão ao endotélio, produção de espécies reativas oxidativas, ativação do sistema inflamatório, aumento da vasoconstrição arteriolar e disfunção na cascata de coagulação. Essas alterações refletem na redução da perfusão placentária e, posteriormente, no surgimento e evolução de distúrbios hipertensivos (FERREIRA MG, et al., 2016; QUEIROZ MR, 2018; QU H e KHALIL R, 2020).

As síndromes hipertensivas intercorrentes na gestação, em especial a pré-eclâmpsia ( $P E)$, acarretam risco real e impacto significativo nos indicadores relacionados à saúde materno-infantil e são a principal causa de morte materna e perinatal no mundo, sendo responsável por $35 \%$ dos óbitos. No Brasil, estima-se que a incidência seja $1,5 \%$ para PE e $0,6 \%$ para eclâmpsia, entretanto sabe-se que esses dados podem estar subestimados e sofrer variações entre as regiões do país (FEBRASGO, 2017). Outro estudo brasileiro, realizado por Giordano JC, et al. (2014), observou que em áreas mais desenvolvidas a prevalência de eclâmpsia foi estimada em $0,2 \%$, com índice de morte materna de 0,8\%, enquanto que em regiões menos favorecidas esta prevalência se eleva para $8,1 \%$ com razão de morte materna podendo chegar a $22 \%$. Reforça-se, ainda, que a maioria dos óbitos relacionados a DHEG poderiam ser evitados se diagnosticados e tratados em tempo hábil (KAHHALE S, et al., 2018).

Vários fatores de risco estão relacionados com o surgimento da DHEG, entre eles os extremos de idade materna, obesidade, antecedentes familiares ou pessoal de PE e presença de patologias crônicas. No entanto, ressalta-se que a maioria dos casos de PE ocorre em mulheres nulíparas saudáveis, sem fatores de risco óbvios e, embora o papel preciso das interações genético-ambientais sobre o risco e a incidência de PE não seja claro, estudos sugerem que possa algum componente genético associado (ACOG, 2020).

A hipertensão gestacional é definida como uma pressão arterial sistólica de $140 \mathrm{mmHg}$ ou mais e/ou uma pressão arterial diastólica de $90 \mathrm{mmHg}$ ou mais, em uma mulher com um quadro previamente normal pressão arterial. A PE é caracterizada pela presença de proteinúria e/ou edema e hipertensão arterial identificada pela primeira vez após a vigésima semana de gestação. Quando diagnosticada em gestantes com hipertensão crônica é denominada de pré-eclâmpsia sobreposta. Mulheres com hipertensão gestacional com variação grave de pressão arterial devem ser diagnosticadas com pré-eclâmpsia com características graves (RAMOS JG, et al., 2017).

Embora a hipertensão e a proteinúria sejam consideradas os critérios clássicos para o diagnóstico de PE, outros critérios também são importantes. Nesse contexto, é recomendado que mulheres com hipertensão gestacional na ausência de proteinúria sejam diagnosticadas com PE se apresentarem características graves como insuficiência hepática, insuficiência renal, trombocitopenia, dor grave persistente no quadrante superior direito ou epigástrica e não explicada por diagnósticos alternativos; edema pulmonar; ou cefaleia de início recente que não responde ao analgésico e não é explicada por diagnósticos alternativos ou distúrbios visuais. Por ter um caráter multissistêmico, a PE pode evoluir para situações mais graves, como a eclâmpsia e a Síndrome de HELLP (PERAÇOLI JL, et al., 2019). 
A eclâmpsia é uma das complicações da PE e é definida pela presença de um episódio de convulsão durante a gestação, no decurso do parto ou no puerpério imediato. Em pacientes com PE, a presença de sinais e sintomas como cefaléia occipital ou frontal, visão borrada, fotofobia e estado mental alterado são sugestivos de eclâmpsia. É fundamental que o diagnóstico seja estabelecido de forma precisa uma vez que pode provocar mortalidade materna e fetal se as condutas que permitam sua reversão forem tardias (ACOG, 2020; FEBRASGO, 2017).

A síndrome de HELLP é outra complicação grave da pré-eclâmpsia, que causa aumento da mortalidade materna e perinatais. A síndrome ocorre entre uma a duas mulheres a cada 1.000 gestações, sendo que a maioria é acometida entre a $28^{\circ}$ a $36^{\circ}$ semana de gestação. Estima-se que a mortalidade materna ocorre em $24 \%$ dos casos. A síndrome se desenvolve de forma súbita e é caracterizada pela presença de anemia hemolítica microangiopática, disfunção hepática e trombocitopenia, por isso o acrônimo HELLP, em que H representa a hemólise, EL os níveis elevados de enzimas hepáticas e LP a contagem baixa de plaquetas (GONÇALVES AP, et al., 2018).

\section{Conduta e tratamento}

O rastreamento de pacientes com PE inclui uma avaliação Doppler das artérias uterinas após a 23a semana de idade gestacional e é útil para avaliar a presença ou não de uma adequada implantação placentária. A predição de pacientes com alto risco de desenvolvimento de PE é feita quando há um fluxo de alta resistência com incisuras protodiastólicas persistentes além de 27 semanas e/ou índice de pulsatilidade alterado (acima do percentil 95 para esta idade gestacional). Além disso, a avaliação laboratorial é de grande importância no rastreamento e inclui a dosagem de bilirrubinas e DHL para rastreio de hemólise, TGO, coagulograma e contagem de plaquetas para rastreio da Síndrome HELLP (FEBRASGO, 2017; WILLIAMS B, et al., 2018).

Uma vez diagnosticada a PE, o objetivo do tratamento é prevenir as complicações maternas e fetais como, por exemplo, descolamento prematuro de placenta, acidente vascular cerebral ou, até mesmo, agravamento do quadro clínico resultando na Síndrome de HELLP e eclâmpsia. O tratamento clínico começa com o repouso relativo, em decúbito lateral esquerdo, que favorece o retorno venoso, aumento do débito cardíaco e fluxo plasmático renal. Além disso, a sedação com o uso de neurolépticos (levomepromazina) na dose de $3 \mathrm{mg}$, por via oral, é importante para diminuir a labilidade emocional (KAHHALE S, et al., 2018).

Orientações dietéticas como dieta hipossódica contendo de dois a três gramas de sal e rica em proteínas são importantes no manejo das pacientes com PE. Para pré-eclâmpsia grave recomenda-se fortemente o tratamento anti-hipertensivo com o intuito de diminuir a morbidade e mortalidade materna e a droga de primeira escolha para o tratamento da crise hipertensiva é a nifedipina (KAHHALE S, et al., 2018).

Todas as gestantes com PE grave devem ser internadas e, no manejo inicial, devem receber sulfato de magnésio e anti-hipertensivos (PAS $\geq 160 \mathrm{mmHg}$ ou PAD $\leq 110 \mathrm{mmHg}$ ). Na PE grave, em uma gestação a termo, com colo favorável para indução do parto, a conduta é realizar o parto. Porém, em mulheres com gestação pré-termo, a antecipação do parto aumenta o risco do recém-nascido ter intercorrências e precisar de internação prolongada, dessa forma, o médico deve ponderar os riscos e benefícios de prolongar a gestação. Em gestações pré-termo, na presença de eclâmpsia, edema pulmonar, coagulopatia e avaliação fetal não reativa, o parto deve ser realizado mesmo antes de completar a corticoterapia para maturidade fetal (RAMOS JG, et al., 2017).

Já a síndrome HELLP é uma emergência obstétrica e requer atendimento imediato. As bases do tratamento são a prevenção das complicações hemorrágicas e da eclâmpsia, o controle da hipertensão arterial grave e o desencadeamento do parto. O manejo da interrupção do parto deve ser realizado de acordo com a gravidade de cada caso. Em gestações superiores a 34 semanas, o parto deve ser induzido e simultaneamente deve ser controlado a crise hipertensiva, administrado sulfato de magnésio e, quando necessário, utilizado hemoderivados. Nas gestantes com menos de 34 semanas, na ausência de complicações graves, como hematoma hepático, plaquetopenia grave e eclâmpsia, deve-se realizar corticoterapia para maturação pulmonar antes de interromper a gestação (RAMOS JG, et al., 2017). 


\section{COVID-19 e doenças hipertensivas na gestação}

No contexto da pandemia do COVID-19, o acompanhamento pré-natal e a monitorização da pressão arterial em ambiente ambulatorial das gestantes foram diminuídos em função de evitar contaminações pelo SARS-CoV-2. Com isso, se tornou de extrema importância o acompanhamento por consultas à distância e a instrução de como realizar a aferição pressórica de forma correta no próprio domicílio, a fim de evitar as complicações da DHEG (BARTON JR, et al., 2020).

Observa-se também um grande número de gestantes infectadas por COVID-19 evoluindo para estados hipertensivos. Acredita-se que essa situação ocorre devido à interação do SARS-CoV-2 com a expressão da enzima conversora de angiotensina 2 (ECA 2), que é uma proteína essencial para a regulação da pressão arterial e está presente em grandes quantidades na placenta. Além de diminuir a expressão da ECA 2, o vírus pode danificar a placenta, iniciando um quadro pré-eclâmpsico. Tal fato pode ser constatado pela maior incidência de partos prematuros e baixo peso ao nascer em pacientes infectadas (ABBAS AM, et al., 2020; JAFARI M, et al., 2021).

Pacientes que já manifestam a pré-eclâmpsia, por compartilharem semelhanças fisiopatogênicas com o COVID-19, possuem maior risco de pior desfecho quando infectadas por esse vírus, com elevada tendência pró-trombótica. Algumas literaturas relataram maiores taxas de morte materna em gestantes infectadas por SARS-CoV-2 com hipertensão crônica pré-existente, entretanto, são necessários mais estudos para constatar a causa direta desses óbitos. Além desse risco mais elevado, a presença de semelhanças nos quadros clínicos da PE e do COVID-19 torna o diagnóstico diferencial e a abordagem adequada mais dificultosos, visto que a PE grave pode simular a piora dessa doença viral (AHMED I, et al., 2020; NARANG K, et al., 2020; ALLOTEY J, et al., 2020).

Outrossim, analisou-se também a presença de uma síndrome com características clínicas semelhantes à PE em pacientes com quadro de infecção por COVID-19 complicados por comprometimento pulmonar. Essa síndrome parece estar ligada a vasoespasmo, alterações plaquetárias, trombose microvascular, "disfunção" endotelial e má perfusão tecidual e pode ser distinguida da PE pela análise das taxas dos fatores angiogênicos relacionados à placenta específicos da insuficiência placentária, como o PIGF e o sFlt-1, do índice de pulsatilidade da artéria uterina (UtAPI) e da lactato desidrogenase (LDH) (MENDOZA M, et al., 2020).

Alguns estudos revelaram, ainda, a presença de trombos intervilosos, que estão relacionados a doença hipertensiva gestacional e correspondem à característica associação do COVID-19 a distúrbios tromboembólicos. Além disso, essas pacientes com enfermidade hipertensiva gestacional e infecção pelo SARS-CoV-2 apresentaram mudanças histopatológicas de má perfusão materna, situação intimamente relacionada com comprometimento do crescimento do feto, nascimento prematuro e óbito fetal (SHANES ED, et al., 2020).

Quanto ao manejo farmacológico da infecção pelo SARS-CoV-2 em gestantes com distúrbios hipertensivos, ainda não existem evidências suficientes disponíveis, visto que essa população não está incluída nos diversos ensaios clínicos destinados a avaliar os possíveis tratamentos para o COVID-19. Dessa forma, se tratando da exposição ao COVID-19, as gestantes devem ser consideradas como grupo vulnerável na população e o contágio deve ser evitado por não saber ao certo as manifestações e consequências desta infecção. Em relação à transmissão do vírus em recém-nascidos, não há evidências concretas significativas da existência da verticalização desta (FOGACCI S, et al., 2020; RONNJE L, et.al., 2020).

Logo, são necessários estudos longitudinais e de base populacional para melhor definição dos riscos da DHEG, associada ou não ao COVID-19, visto que a identificação e controle antecipado reduz o impacto das possíveis intercorrências gestacionais (JACOB LS, et. al., 2021; NARANG K et al., 2020). Reforça-se, ainda, que os cuidados necessários para prevenir as complicações da DHEG se estendem desde os cuidados no pré-natal aos cuidados pós-parto, incluindo o controle da PA no puerpério. Além disso, a realização de medidas preventivas e assistenciais, na maioria das vezes com protocolos simples e pouca tecnologia de recursos, pode refletir positivamente, logrando êxito ao objetivo de promover e preservar a saúde maternoinfantil (OLIVEIRA AM e GRACILIANO NG, 2015; BROWN MA, et. al.,2018). 


\section{CONSIDERAÇÕES FINAIS}

O presente trabalho mostrou a importância do cuidado pré-natal para a saúde materna e fetal, de forma a permitir a detecção precoce de doenças, entre elas a DHEG, bem como o tratamento adequado e a prevenção de complicações, como a eclampsia e a síndrome de HELLP. Deve-se ainda reforçar os cuidados frente à pandemia do COVID-19 uma vez que se tem observado a interferência da infecção pelo coronavírus no aumento do número e da gravidade das síndromes hipertensivas gestacionais. Dessa forma, vale ressaltar que, mesmo durante o atual momento de pandemia requerendo isolamento social, é de extrema importância que as gestantes e as equipes de saúde planejem a melhor forma de assistência pré-natal, considerando a telemedicina sempre que sua presença física puder ser evitada no consultório médico.

\section{REFERÊNCIAS}

1. ABBAS AM, et al. COVID-19 and maternal pre-eclampsia: A synopsis. Scandinavian journal of immunology, 2020; 92(3): e12918.

2. ACOG. Gestational Hypertension and Preeclampsia - Practice Bulletins of the American College of Obstetricians and Gynecologists. Obstetrics \& Gynecology, 2020;135(6):e237.

3. AHMED I, et al. Severe pre-eclampsia complicated by acute fatty liver disease of pregnancy, HELLP syndrome and acute kidney injury following SARS-CoV-2 infection. BMJ case reports, 2020; 13(8): e237521.

4. ALLOTEY J, et al. Clinical manifestations, risk factors, and maternal and perinatal outcomes of coronavirus disease 2019 in pregnancy: living systematic review and meta-analysis. British Medical Journal, 2020; 1;370:m3320

5. BARTON JR, et al. A Proposed Plan for Prenatal Care to Minimize Risks of COVID-19 to Patients and Providers: Focus on Hypertensive Disorders of Pregnancy. American Journal of Perinatology, 2020; 37(8):837-844.

6. BROWN MA, et al. The hypertensive disorders of pregnancy: ISSHP classification, diagnosis \& management recommendations for international practice. Hypertension, 2018;72(1):24-43.

7. FEBRASGO. Pré-eclâmpsia nos seus diversos aspectos - Federação Brasileira das Associações de Ginecologia e Obstetrícia. 2017. Disponível em: https://www.febrasgo.org.br/media/k2/attachments/12-PRE_ECLAyMPSIA.pdf. Acessado em: 21 de março de 2021.

8. FERREIRA MG, et al. Nursing care for women with pre-eclampsia and/or eclampsia: integrative review. Revista da Escola de Enfermagem da USP, 2016; 50(2):324-334.

9. FOGACCI S, et al. Management of pregnancy-related hypertensive disorders in patients infected with SARS CoV-2: pharmacological and clinical issues. European Heart Journal - Cardiovascular Pharmacotherapy, 2020; pvaa105.

10. GIORDANO JC, et al. The burden of eclampsia: results from a multicenter study on surveillance of severe maternal morbidity in Brazil. PLoS One. 2014;9(5):e97401.

11. GONÇALVES AP, et al. Síndrome de HELLP: Entendendo e intervindo. Revista Saúde em Foco, 2018; $10: 274-278$.

12. JACOB LS, et al. Instrumento sobre conhecimento, atitude e prática de gestantes acerca da síndrome hipertensiva gestacional. Revista Rene (Online), 2021; 22: e60040.

13. JAFARI M, et al. Clinical characteristics and outcomes of pregnant women with COVID-19 and comparison with control patients: A systematic review and meta-analysis. Reviews in Medical Virology, 2021; e2208.

14. KAHHALE S, et al. Pré-eclâmpsia. Revista de Medicina, 2018;97(2):226-34.

15. KARIMI L, et al. Effect of COVID-19 on Mortality of Pregnant and Postpartum Women: A Systematic Review and Meta-Analysis. Journal of Pregnancy, 2021:1-33.

16. MÉHATS C, et al. New perspectives on pre-eclampsia. Médecine/sciences (Paris), 2017;33(12):1079-1088.

17. MENDOZA M, et al. Pre-eclampsia-like syndrome induced by severe COVID-19: a prospective observational study. British Journal Of Obstetrics and Gynaecology, 2020; 127(11):1374-1380.

18. NARANG K, et al. SARS-CoV-2 Infection and COVID-19 During Pregnancy: A Multidisciplinary Review. Mayo Clinic proceeding, 2020; 95(8):1750-1765.

19. OLIVEIRA AM, GRACILLIANO NG. Síndrome hipertensiva da gravidez e diabetes mellitus gestacional em uma maternidade pública de uma capital do Nordeste brasileiro, 2013: prevalência e fatores associados. Epidemiologia Serviço Saúde, 2015;24(3):441-451.

20. OMS. Recomendações da OMS para a Prevenção e tratamento da pré-eclâmpsia e da eclâmpsia. 2014. Disponível em: https://www.who.int/reproductivehealth/publications/maternal_perinatal_health/. Acessado em: 25 de março de 2021.

21. PERAÇOLI JL, et al. Pré-eclampsia e eclampsia. Revista Brasileira de Ginecologia e Obstetrícia, 2019; 41(5):318332.

22. QUEIROZ MR. Síndromes hipertensivas na gestação no Brasil: estudo a partir dos dados da pesquisa "Nascer no Brasil. Tese (Doutorado em Saúde, Ciclos de Vida e Sociedade) - Faculdade de Saúde Pública, Universidade de São Paulo, São Paulo, 2018; 66 p.

23. QU H, KHALIL RA. Vascular mechanisms and molecular targets in hypertensive pregnancy and preeclampsia. American Journal of Physiology-Heart and Circulatory Physiology, 2020; 319(3):661-681.

24. RAMOS JG, et al. Preeclampsia. Revista Brasileira de Ginecologia e Obstetrícia, 2017; 39(9): 496- 512.

25. RIBEIRO JF, et al. Hellp Syndrome: Obstetric Characterization and Treatment Modality. Journal of Nursing, 2017;11(3):1343-1348.

26. RONNJE L, et al. Complicated COVID-19 in pregnancy: a case report with severe liver and coagulation dysfunction promptly improved by delivery. BMC Pregnancy Childbirth, 2020; 20(1):511.

27. SHANES ED, et al. Placental Pathology in COVID-19. American Journal of Clinical Pathology, $2020 ; 154(1): 23-32$.

28. SOUSA MG, et al. Epidemiology of artherial hypertension in pregnants. Einstein, 2020; 18: eAO4682. 